\title{
EFFECTS OF REACTION TIME PERFORMANCE ON SINGLE- UNIT ACTIVITY IN THE CENTRAL AUDITORY PATHWAY OF THE RHESUS MACAQUE ${ }^{1}$
}

\author{
ALLEN F. RYAN, ${ }^{* 2}$ JOSEF M. MILLER, $\$$ BRYAN E. PFINGST, $\ddagger$ AND GLEN K. MARTIN $\ddagger$ \\ * Division of Otolaryngology, Department of Surgery, University of California at San Diego, La Jolla, California 92039 and \\ $\ddagger$ Department of Otolaryngology/Head and Neck Surgery and Regional Primate Research Center, University of Washington, \\ Seattle, Washington 98195
}

Received March 21, 1983; Revised September 7, 1983; Accepted September 13, 1983

\begin{abstract}
The activity of single units at various locations in the central auditory pathway of rhesus macaques was recorded during the monkeys' performance and nonperformance in an auditory reaction time task. Evoked unit responses during performance were compared with those observed during passive delivery of identical stimuli. Single units were recorded from the cochlear nucleus, superior olivary complex, lateral lemniscus, inferior colliculus, medial geniculate nucleus, and auditory cortex. Significant effects of task performance on unit discharge patterns were observed at all levels of the central auditory pathway: Spontaneous discharge rates in the more peripheral auditory nuclei tended to be higher during performance. Evoked discharge that occurred relatively late during a stimulus presentation ( $>75 \mathrm{msec}$ after stimulus onset) was increased during performance, compared with the nonperformance condition, in nuclei above the cochlear nucleus. The initial latency of evoked discharge was increased during performance for subcortical nuclei but was decreased for units in auditory cortex. These results suggest that the effects of performance may be mediated by a tonic increase in the excitability of auditory units which operates primarily at peripheral auditory stations, and a descending, stimulus-evoked increase in excitability which primarily influences the cells of higher auditory nuclei. At the cortical level, these changes lead to increased signal-to-noise ratio of the evoked response during performance in the auditory task.
\end{abstract}

The response properties of single units in the central auditory pathway have been studied extensively in the acute, anesthetized preparation. Over the years, it has become increasingly clear that the responses of auditory neurons can vary widely, depending on such factors as anesthetic state (Erulkar et al., 1956; Evans and Nelson, 1973), state of arousal (Hubel et al., 1959; Pfingst et al., 1977), and training and performance in a behavioral task (Holstein et al., 1969a, b; Miller et al., 1972, 1982; Beaton and Miller, 1975; Woody et al., 1977).

In previous investigations, we have observed significant effects of performance in a reaction time task on the evoked response properties of single units at the levels of the auditory cortex (Miller et al., 1972; Beaton

\footnotetext{
${ }^{1}$ This work was supported by National Institutes of Health Grants NS08181, RR00166, and NS00176. We express appreciation to Judy Miller for histological preparation and to Kate Schmitt for editorial assistance.

${ }^{2}$ To whom correspondence should be addressed, at Division of Otolaryngology, Department of Surgery, University of California at San Diego, La Jolla, CA 92093.
}

and Miller, 1975; Pfingst et al., 1977) and inferior colliculus (Ryan and Miller, 1977). These observations raise questions concerning ( 1 ) the generality of performance effects on auditory responsiveness throughout the auditory pathway, and (2) the mechanisms that underlie these performance-dependent changes. The presence or absence and the characteristics of performance-related differences along the auditory pathway may provide insight into the origins of behaviorally dependent changes in auditory signal processing. The central auditory pathway has not been surveyed systematically at the singleunit level. Previous investigations using gross evoked potentials or multiunit recordings have led to conflicting conclusions regarding the generality of arousal and attentional effects on evoked responsiveness. While several investigators observed significant effects throughout the central auditory pathway (Bogdanski and Galambos, 1960; Worden and Marsh, 1963; Chin et al., 1965; Oleson et al., 1975), others have seen changes only at auditory cortex and medial geniculate nucleus (Winters, 1964; Wickelgren, 1968). Single-unit studies may clarify this 
issue and lead to a better understanding of the mechanisms involved.

\section{Materials and Methods}

Subjects. Subjects were 12 adolescent male rhesus macaques (Macaca mulatta) weighing 2.5 to $4.0 \mathrm{~kg}$. Experimental sessions were conducted in a double-walled, sound-attenuated chamber (IAC 1200A). All behavioral and auditory control equipment was located outside the experimental chamber. Subjects were restrained in a standard primate chair during the sessions and were caged individually at all other times.

Sound generation and control. White noise was provided by a random noise generator (General Radio 1381) with a bandwidth of $20 \mathrm{~Hz}$ to $50 \mathrm{kHz}$. Pure tones were generated by a beat-frequency oscillator (Hewlett Packard 202). Tone pulses were formed with 5 -msec rise-fall times by a tone switch (Grason-Stadler). The envelope of the stimulus was applied to an attenuator pad (Hewlett Packard 350D), which permitted attenuation of the signal in 1-dB steps, and was delivered through dynamic ear speakers (TDH 49 or Beyer DT-48) mounted in a rubber cuff that fit over the external ear.

Sound pressure levels were measured by a wave analyzer (General Radio 1900A) and a $1 / 2$-inch condenser microphone (Brüel and Kjaer) fitted with a calibrated probe tube that was inserted through a hole in the circumaural cuff and terminated at the opening of the external auditory meatus. Further details of the sound presentation and delivery system have been described elsewhere (Pfingst et al., 1975a).

Behavioral procedures. The procedures used for training and testing animals in the reaction time task have been described previously (Stebbins and Miller, 1964; Pfingst et al., $1975 \mathrm{a}, \mathrm{b})$. Briefly, the animals were shaped in a series of small steps to perform the following task. At the onset of a visual alerting stimulus, they depressed a telegraph key and held it down for a variable period (1 to $4 \mathrm{sec}$ ) until the onset of a fixed-duration auditory stimulus ( 200,250 , or $325 \mathrm{msec}$ ), after which they quickly released the key to obtain applesauce reinforcement. The reward was delivered only for key releases with latencies of 0.1 to $1.0 \mathrm{sec}$ after stimulus onset. Key release was followed by a 2.0 -sec response-free period before onset of the next visual alerting stimulus. Response latencies of $<0.1 \mathrm{sec}$ or $>1.0 \mathrm{sec}$ initiated the intertrial interval, and the resulting delay in reinforcement suppressed inappropriate response to an average level of less than $5 \%$ of all bar presses. (It should be noted that we seldom used stimuli near threshold intensity in this investigation.)

The animals also were adapted to periods during which acoustic stimuli were presented repetitively in the $a b$ sence of reinforcement. The mean rate at which stimuli were presented was comparable to that during the performance period. The monkeys quickly stopped responding during these nonperformance intervals. In both the performance and nonperformance situations, the stimuli included white noise and a variety of pure tones.

In a few early subjects, duration of the stimulus in the performance condition was controlled by the animal's reaction time, i.e., stimulus termination coincided with key release, typically between 200 and $500 \mathrm{msec}$. Thus, stimulus duration in these subjects was variable and increased with decreasing stimulus intensity. In most subjects used in this study, constant-duration stimuli were used.

Surgical procedures. After behavioral training, the animals underwent aseptic surgery for implantation of a single-unit recording chamber. Each subject was given a presurgical dose of Vetalar, intubated, anesthetized with halothane vapor, and placed in a Kopf stereotaxic frame. A skin flap was reflected, and a circle of bone $16 \mathrm{~mm}$ in diameter was removed from a location consistent with the stereotaxic position of auditory structures to be studied. The stereotaxic coordinates for brain stem and midbrain structures in the sitting $M$. mulatta were based on the atlas of Smith et al. (1972). Coordinates for location of auditory cortex and medial geniculate nucleus were developed empirically. A stainless steel chamber (15 to $36 \mathrm{~mm}$ high, $16 \mathrm{~mm}$ outer diameter, $13 \mathrm{~mm}$ inner diameter) was implanted over the bone defect. In some animals the chamber was oriented in the coronal stereotaxic plane and directed toward the midbrain and brain stem with a $17^{\circ}$ lateral angle. In others, a direct vertical approach was employed. The end of the chamber was advanced just until it contacted the dura. The base of the chamber was sealed with a layer of silicone rubber, which maintained a closed system in the chronic preparation. Stainless steel screws were threaded into the bone, and the chamber was cemented to the skull and screws with dental acrylic. A device that allowed rigid stabilization of the head also was implanted at this time. For further details, see Miller and Sutton (1976) and Pfingst and O'Connor (1980).

Electrophysiological recording. Once the animals had recovered from surgery, behavioral sessions were resumed, this time with head restraint. Animals adapted easily to the restraint and resumed normal behavior in the reaction time procedure within 1 to 4 days. Then neural recording was initiated. The procedures used to record single units along the auditory pathway in the chronic monkey have been described in detail elsewhere (Miller and Sutton, 1976; Ryan and Miller, 1977, 1978; Pfingst and O'Connor, 1980, 1981; Lonsbury-Martin and Martin, 1981). Briefly, an adjustable guide and coupler were mounted on the implanted chamber, which provided access to an area delimited by a $5-\mathrm{mm}$ radius from the center of the chamber. A sterile 21 gauge needle cannula was attached to a hydraulic microdrive (Trent-Wells). A tungsten microelectrode, insulated with Epoxylite and, in some cases, tipped with an electroplated iron ball of 4 to $25 \mu \mathrm{m}$ diameter, was inserted into the cannula. The micromanipulator was inserted into the adjustable guide, which directed the cannula into the brain. Cannula length was selected to terminate 6 to $10 \mathrm{~mm}$ above the target structure. The electrode was advanced out of the cannula with the hydraulic microdrive. White noise bursts with an intensity of 80 to $90 \mathrm{~dB}$ sound pressure level, delivered in the nonperformance condition, were routinely used as searching stimuli until the first responses were evoked on a given track. Then single units were tested with white noise and pure tones for the 
remainder of the track. On the most effective electrode penetrations, marking lesions $(40 \mu \mathrm{A}$ for 15 to $30 \mathrm{sec})$ were made with a DC constant-current lesion maker (Grass LM54).

The signal from the microelectrode was amplified by a unity-gain FET follower and a preamplifier (Grass P511F), passed through a high pass $(>0.5 \mathrm{kHz})$ filter, and monitored on an oscilloscope (Tektronix RM565) and an audio monitor (Grass AM8). The oscilloscope was internally triggered from single-unit discharges. Trigger gate pulses from the oscilloscope were applied to the input of a dot raster system. The output pulses from the raster, along with the filtered electrode output and the acoustic stimulus, were displayed on a memory oscilloscope (Tektronix 564), which was triggered by onset pulses to the tone switch.

The neuronal discharge also was recorded on one channel of an eight-track FM tape recorder (Honeywell 5600). The onset and offset pulses from the tone switch, as well as the acoustic stimuli, were recorded on other channels.

When a single unit that was responsive to the acoustic stimulus was isolated, it was examined by presenting short, alternating blocks of performance and nonperformance trials. The acoustic stimuli were either white noise or a pure tone at the unit's best frequency, presented either at a variety of intensities or at the unit's dominant intensity. Characteristic frequency was determined by successive discrete frequency sweeps at decreasing intensities. Performance and nonperformance stimuli were of the same fixed duration except for the performance stimuli of some early subjects mentioned above. Intensity levels were presented in a pseudorandom order within each block of performance and nonperformance trials, which varied from 10 to 40 stimulus presentations. Transition from performance to nonperformance conditions was signaled by failure of the visual alerting stimulus to appear, and from nonperformance to performance it was signaled by reappearance of the alerting stimulus.

Data analysis. All data were analyzed from the taped recordings of single-unit activity. Units were isolated and digitized with the internal trigger of the RM565 oscilloscope. Isolation was based primarily on spike amplitude and waveform. Only data from clear, stably isolated units were included in this study. Since alterations in waveform or marked changes in the spontaneous activity (not associated with a change in behavioral state) are possibly indicative of damage or neurophysiological changes in a unit, such changes led to cessation of study or elimination of a unit from the study. Statistical analyses of initial latencies and of firing rates for selected intervals, as well as the generation of peristimulus time histograms, were performed by a Prime 200 computer. For units with high spontaneous rates, initial latencies were determined by eye from high resolution dot rasters. The majority of units were excitatory and exhibited either an initial phasic increase in firing rate associated with stimulus onset, a sustained response, or both. Phasic responses, when present, lasted for 50 to $75 \mathrm{msec}$. Therefore, two analysis intervals were selected to include the periods 0 to $75 \mathrm{msec}$ and 75 to $200 \mathrm{msec}$ after stimulus onset. This permitted independent evaluation of the phasic and sus- tained components of unit responses. In comparing evoked unit activity in the performance and nonperformance conditions, all trials collected under the same stimulus conditions were included, with one exception. A few units exhibited obvious variation in activity on the first few trials following a change in performance conditions. In such units, analysis included only later trials in each condition in which response characteristics of the unit had stabilized. Such determination of sequential changes in evoked firing rates and/or patterns within a given block of stimulus trials were made visually from dot raster displays.

Histology. After 8 to 30 days of neural recording, animals were deeply anesthetized with sodium pentobarbital and perfused intracardially with normal saline solution, followed by a $10 \%$ buffered formalin solution. The top of the skull was removed, and the animal was placed in a stereotaxic frame in the same position as that used to orient the recording chamber. $\Lambda$ knife blade was attached to an electrode carrier and was used to cut a thick coronal section of tissue parallel to and containing all electrode tracts. The tissue block was frozen sectioned at $30 \mu \mathrm{m}$, and alternate sections were stained with cresyl violet. Identification of recording sites was made from these sections on the basis of electrode tracks and marking lesions.

\section{Results}

\section{Behavior}

The monkeys' performance in the reaction time task resembled that of humans in a simple, auditory reaction time task (Pfingst et al., 1975a, b). Minimum latencies to intense stimuli were stable for a given animal and varied from about 180 to $350 \mathrm{msec}$ among animals. In general, subjects responded promptly and consistently within the short blocks of performance trials, which were separated by similar blocks of nonperformance trials, until they became satiated. At satiation, responses at the end of a nonperformance block were initiated slowly, if at all. At this time the session was ended.

\section{Recording sites}

Performance and nonperformance data were obtained from 212 units in the 12 subjects. These included 22 units in the central region of the ventral cochlear nucleus, a broad region of primarily multipolar cells; 5 units in the superior olivary complex ( 3 in the medial superior olive and 2 in the periolivary nucleus); 49 in the nuclei of the lateral lemniscus ( 35 in the dorsal nucleus and 14 in the ventral nucleus); 80 units in the inferior colliculus (56 in the central nucleus, 12 in the external capsule, and 12 unspecified); 32 units in the medial geniculate nucleus $^{3} ; 21$ units in the auditory cortex (8 in area A1; 7

\footnotetext{
${ }^{3}$ The medial geniculate nucleus has consistently proven to be the most difficult structure from which to isolate and record auditory units in the unanesthetized monkey. While the position of 32 units could be defined as being within the medial geniculate nucleus, the number of penetrations necessary to locate this structure and to record the appropriate number of units from a given subject made precise specification of recording site in the various medial geniculate nucleus subdivisions impossible.
} 
in belt areas CM, L, and RL; and 6 on the border of A1 and one of the belt areas (Merzenich and Brugge, 1973; Pandya and Sanides, 1973)); and 3 units in motor areas ( 1 in the motor nucleus of the trigeminal nerve and 2 in the pyramidal tract). The discharge patterns of the latter units showed a relation to auditory stimuli, but only in the performance condition.

Figure 1 shows the location of 68 brain stem units recorded from two subjects, including 10 in the inferior colliculus, 49 in the lateral lemniscus, 5 in the superior olivary complex, 1 in the cochlear nucleus, and 3 in motor areas.

\section{Effects of reaction time performance on unit discharge characteristics}

Evoked discharge rate. The most commonly observed effect of reaction time performance on unit activity was an increase in evoked discharge (Figs. 2 and 3). In most cases, this increase was relatively modest for the first 50 to $75 \mathrm{msec}$ of the evoked response, and it was greater from about 75 msec after stimulus onset to the end of the evoked response.

Figure 2 shows the responses of a unit in the dorsal nucleus of the lateral lemniscus to a pure tone at the

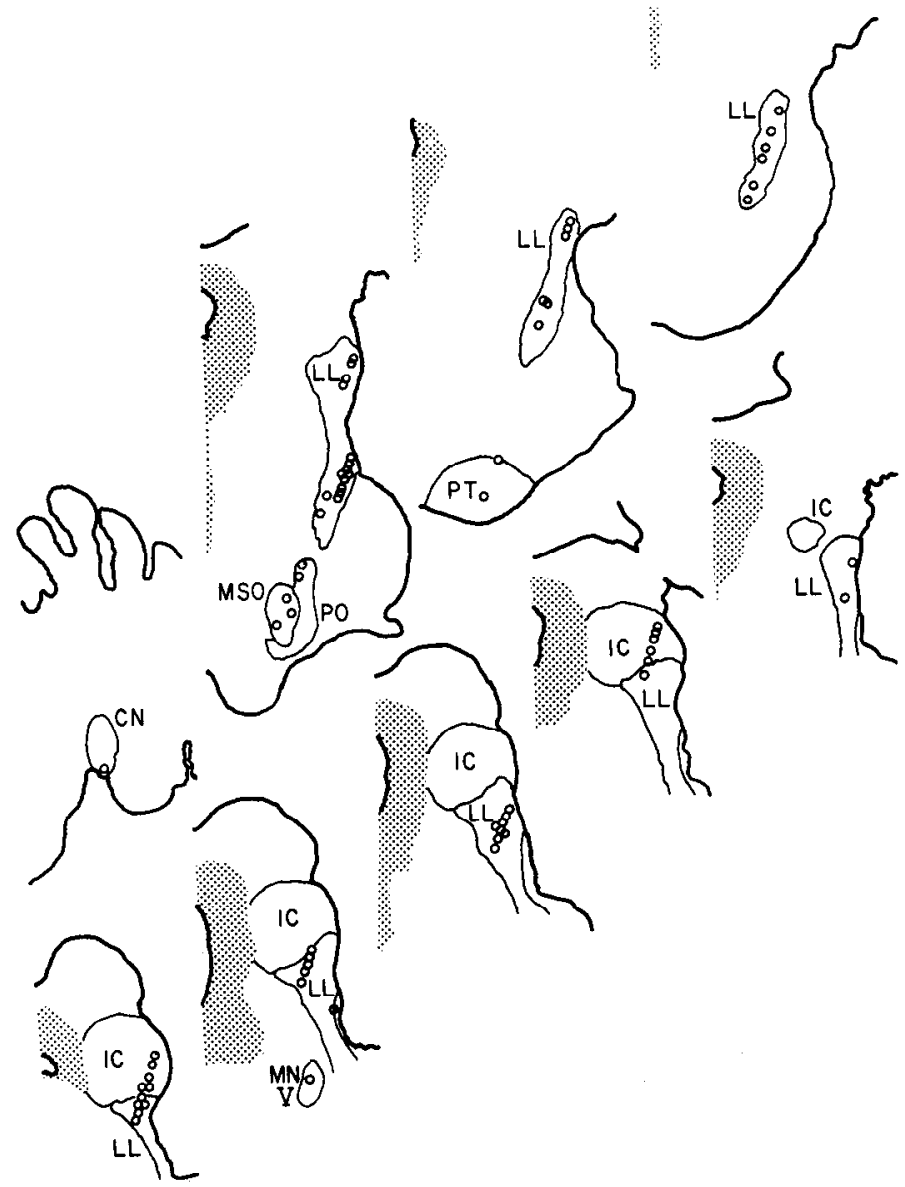

Figure 1. Camera lucida drawings showing locations of 68 units in two subjects. Diagonal groupings represent separate animals. $C N$, cochlear nucleus; $I C$, inferior colliculus; $L L$, lateral lemniscus; $M N V$, motor nucleus of the trigeminal nerve; $M S O$, medial nucleus of the superior olivary complex; $P O$, periolivary nucleus; $P T$, pyramidal tract. unit's characteristic frequency, at various intensities. The dot rasters demonstrate that evoked discharge during performance was greater than that during nonperformance stimulus delivery. The increase was relatively modest during the phasic onset and was more dramatic after the onset response. The preresponse interval showed no significant effect of performance on the unit's spontaneous discharge. Figure 3 compares intensity functions obtained in the performance and nonperformance conditions from a unit in auditory cortex. Evoked discharge in the first $75 \mathrm{msec}$ of the evoked response was greater during performance than during nonperformance at all intensites. A somewhat greater performance-related increase was seen between 75 and $200 \mathrm{msec}$. Reaction time performance had no effect on the spontaneous rate of this unit.

Discharge rates in the two behavioral conditions were compared statistically across all 212 units. While spontaneous rates of discharge and evoked discharge in the first $75 \mathrm{msec}$ of the response for the two conditions were not significantly different, evoked discharge between 75 and $200 \mathrm{msec}$ after the onset of the response was significantly higher ( $p<0.05$, paired $t$ test) during performance than during nonperformance.

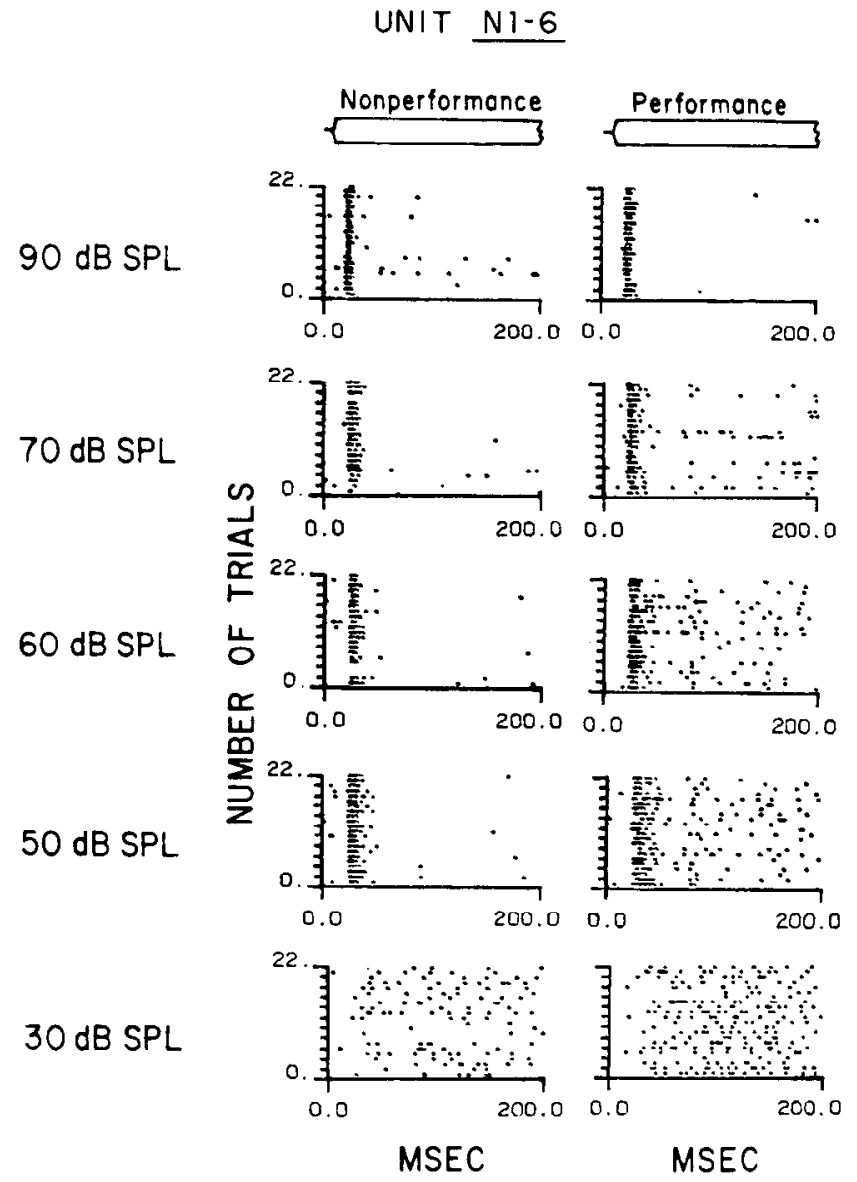

Figure 2. Dot rasters showing the effect of reaction time performance on the discharge pattern of unit N1-6 in the dorsal nucleus of the lateral lemniscus. Each dot raster display represents 22 presentations of the white noise stimulus. The onset response was slightly enhanced during performance, and the following discharge much greater in the performance condition for all but the most intense stimuli. 


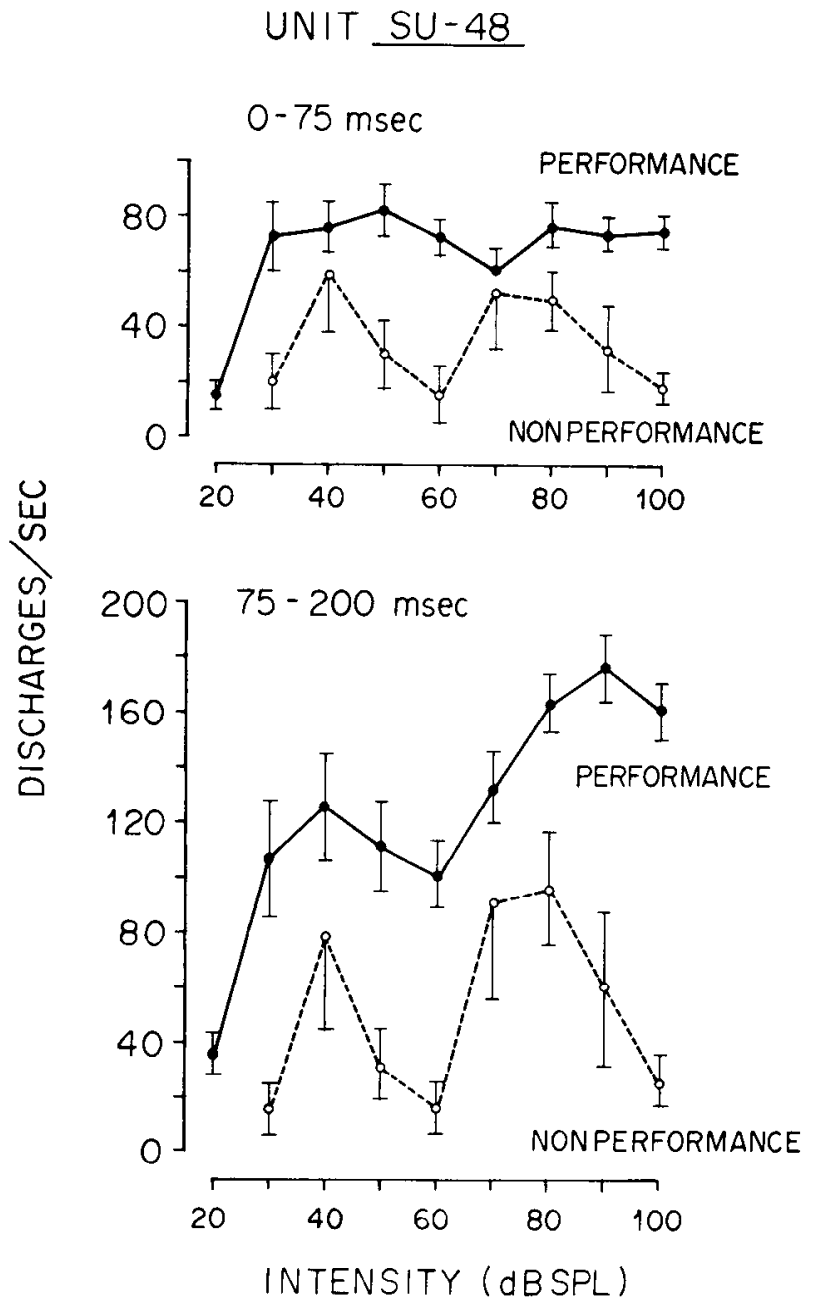

Figure 3. Evoked discharge rate of unit SU-48 in auditory cortex during performance and nonperformance. Each point represents the mean of 20 stimulus presentations; vertical bars represent $1 \mathrm{SD}$.

Initial latency. In subcortical nuclei, the initial latency of evoked discharge increased during the performance condition. The amount of increase was larger with each ascending auditory nucleus up to and including the medial geniculate nucleus. Figure 4 compares latency-intensity functions obtained in the performance and nonperformance conditions for a unit in the medial geniculate nucleus. Initial latency was markedly increased during performance. This difference was greatest at the lowest intensity presented, and it decreased regularly with increasing stimulus intensity, a pattern that was observed regularly at levels below auditory cortex.

In auditory cortex, initial latencies of evoked unit response during reaction time performance were consistently decreased relative to the nonperformance condition (Fig. 5). For the representative unit in Figure 5, task performance was associated with shorter initial latencies at all intensities. The latency change for these cortical units, unlike that for the subcortical units, remained constant across intensities.

Initial latency at the stimulus intensity that evoked maximal discharge was examined statistically for all units. Initial latencies increased during reaction time

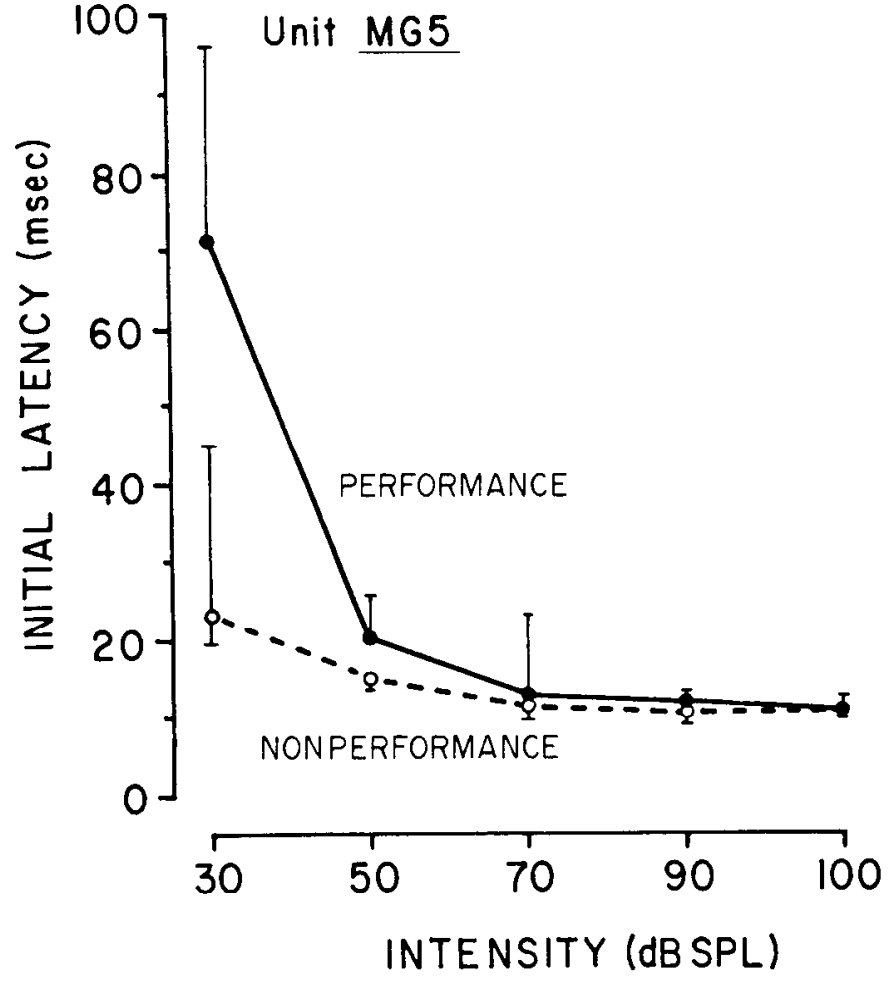

Figure 4. Effect of reaction time performance on the initial latency of unit MG5, in the medial geniculate nucleus, to white noise stimulus. Points represent median latency; bars represent interquartile ranges.

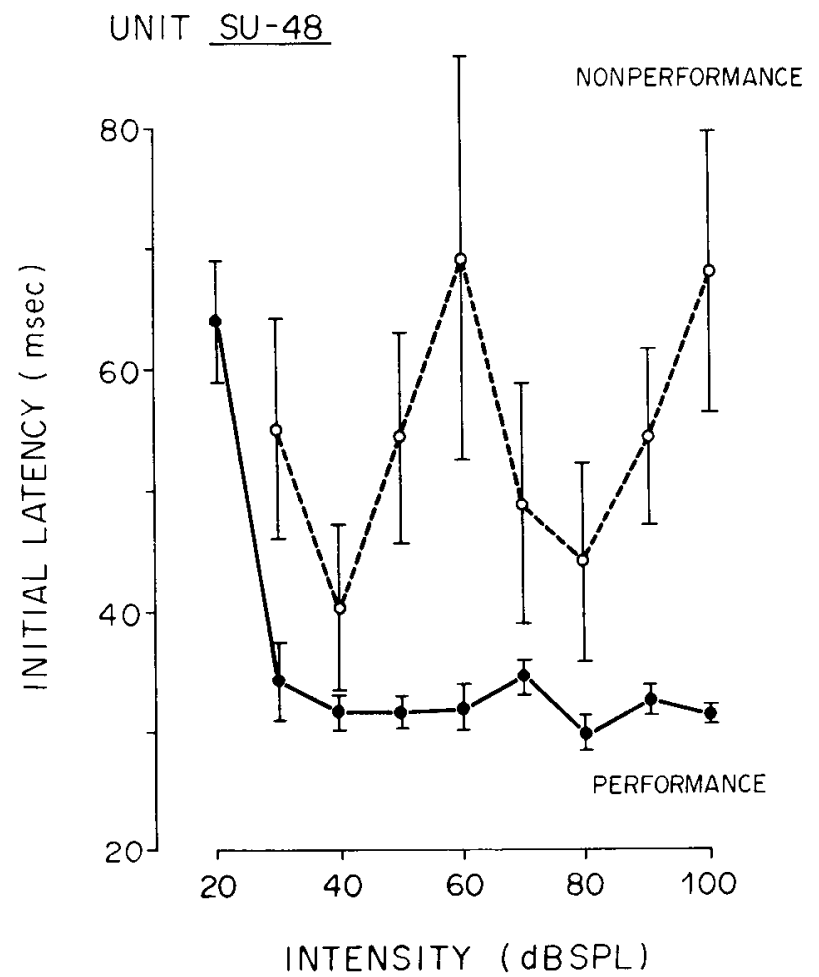

Figure 5. Effect of reaction time performance on initial latency of unit SU-48 in auditory cortex. Initial latencies across a wide range of stimulus intensities were both longer and more variable in the nonperformance condition. Each point represents the mean of 20 stimulus presentations; vertical bars represent $1 \mathrm{SD}$. 
performance in $70 \%$ of all subcortical units, with the remaining units showing a decrease or no effect. The mean effect of performance on initial latency across all subcortical units was an increase of $1.4 \mathrm{msec}(p<0.05$, paired $t$ test). Initial latencies decreased in $62 \%$ of cortical units, and the mean effect across all units was a decrease of 1.8 msec $(p<0.05)$.

Spontaneous rate. Figure 6 shows mean spontaneous rates recorded in the performance and nonperformance conditions for the 209 auditory units included in this report. This rate decreased in a regular fashion at successively higher auditory stations, but within a station only small differences were seen between the performance and nonperformance conditions.

\section{Additional effects of reaction time performance on unit discharge}

In addition to the general effects of reaction time performance described above, occasionally we observed specific, temporally discrete effects on unit discharge that were related to performance. In the inferior colliculus, performance was associated with suppression of offset responses if stimulus duration exceeded 150 to 200 msec, as we have previously reported (Ryan and Miller, 1977). Figure 7 illustrates another effect of reaction time performance, also observed in the inferior colliculus. This unit displayed a brief period of discharge suppression late in the evoked response, only during task performance. The period of discharge suppression occurred with a latency that depended on stimulus intensity, although not in the same manner as the initial latency of the evoked response. The latencies of the late response suppression behaved in a manner more similar to the animal's reaction times, but they were about $200 \mathrm{msec}$ shorter than the reaction times.

\section{Comparison of stations in the auditory pathway}

The effects of performance upon discharge characteristics were evaluated statistically for each unit, using an unpaired $t$ test and a criterion of $p<0.05$. Comparisons for evoked responses were obtained at the unit's best intensity. The results of this analysis are presented in Figure 8. About $34 \%$ of all units showed significant effects of performance on evoked discharge between 0 and $75 \mathrm{msec}$ after response onset, whereas $43 \%$ of units showed such effects on evoked discharge between 75 and 200 msec after response onset. The proportion of significant effects on evoked discharge was relatively constant across stations. About $22 \%$ of all units exhibited initial latencies that were significantly different between the two behavioral conditions. The proportion of units showing significant effects on latency increased at higher auditory centers. Statistically significant effects of reaction time performance on spontaneous discharge rate were observed in $21 \%$ of all units. The proportion of units showing significant effects on spontaneous rate was higher at the peripheral auditory nuclei and decreased sharply at higher auditory stations.

To allow comparison of the effects of reaction time across units with widely varying discharge rates, we

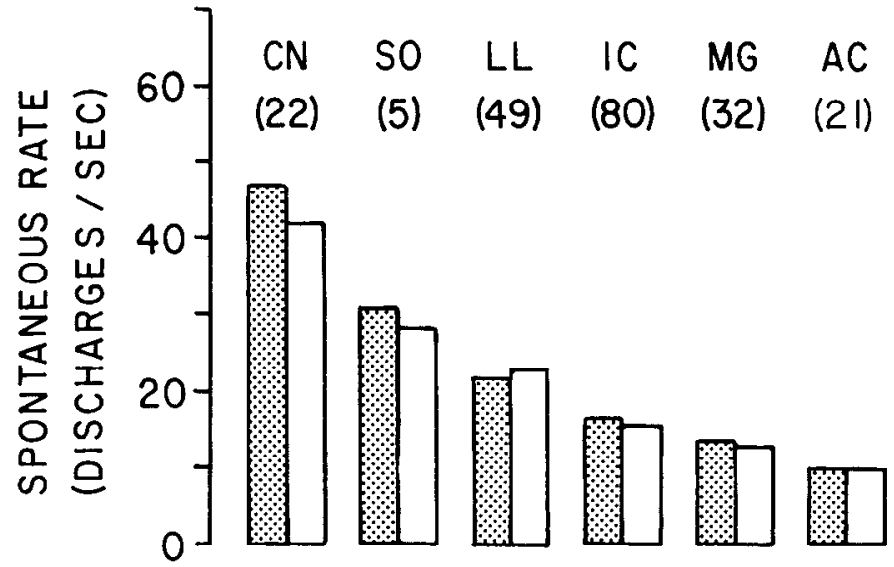

Figure 6. Average spontaneous discharge rate at various locations in the central auditory pathway. The number of units in each sample is indicated in parentheses. Mean rate in the performance condition is indicated by the stippled bar, and in the nonperformance condition by the open bar. $C N$, cochlear nucleus; $S O$, superior olivary complex; $L L$, lateral lemniscus; $I C$, inferior colliculus; $M G$, medial geniculate nucleus; $A C$, auditory cortex.

normalized the rates as follows:

$$
\frac{\text { Performance rate }- \text { Nonperformance rate }}{(\text { Performance rate }+ \text { Nonperformance rate }) / 2}
$$

The resultant values represent the percentage increase or decrease of discharge rate during reaction time performance. The results of this analysis for all units (open bars), as well as for only those units that displayed significant performance-related differences in response parameters as defined above (solid bars), are presented in Figure 9.

Evoked discharge rate. Across all levels of the pathway, evoked discharge rate from 0 to $75 \mathrm{msec}$ after stimulus onset increased by about $10 \%$ during performance. This increase was greatest for units in auditory cortex and least for units in the inferior colliculus and medial geniculate nucleus.

Evoked discharge between 75 and $200 \mathrm{msec}$ after stimulus onset increased about $25 \%$ across all units. This increase was greatest for higher auditory nuclei. When only units that showed a significant difference in discharge rate during this period were considered, this increase averaged about $40 \%$ for all stations above the cochlear nucleus.

Initial latency. As discussed above, initial latencies showed a strong tendency to increase during performance in subcortical units. The average change in latency across all units within a structure increased regularly from about $1.1 \mathrm{msec}$ in the cochlear nucleus to $3.1 \mathrm{msec}$ in the medial geniculate nucleus. When only units showing a significant difference between the two conditions were considered, the subcortical latency increase averaged 4 to $5 \mathrm{msec}$ at all stations. This indicates that the increasing average latency difference seen for all units at successively higher auditory nuclei reflects the increasing proportion of units showing a significant increase in this response parameter at higher stations.

In auditory cortex, initial latencies showed an average 

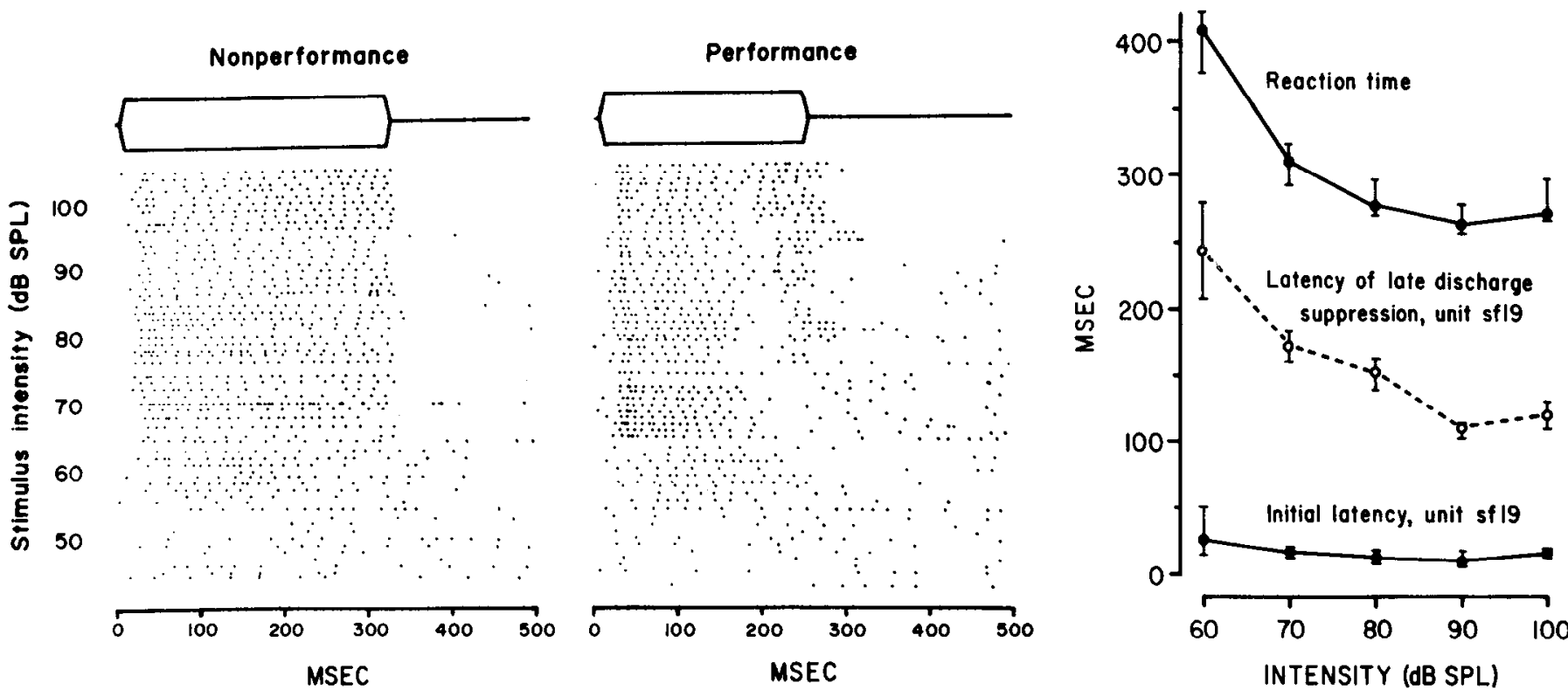

Figure 7. Effect of reaction time performance on the discharge characteristics of unit SF-19 in the inferior colliculus. Duration of the white noise stimulus in the performance condition was controlled by the subject's reaction times. The stimulus duration indicated above the performance dot raster display is the shortest stimulus observed for the trials presented in this figure. Note the gap in the otherwise sustained excitatory discharge pattern, seen only during performance. (The late discharge at 80 to 90 $\mathrm{dB}$ sound pressure level is the resumption of the sustained excitatory response, not an offset response.) The graph at the right illustrates the relation between the latency of the late discharge suppression seen in the dot raster under the performance condition, and both the initial latency of the unit and the subject's reaction times. The latency of the late suppression more closely resembles the reaction times. Each point in the figure represents a median latency of reaction time. The vertical hars represent interquartile ranges.

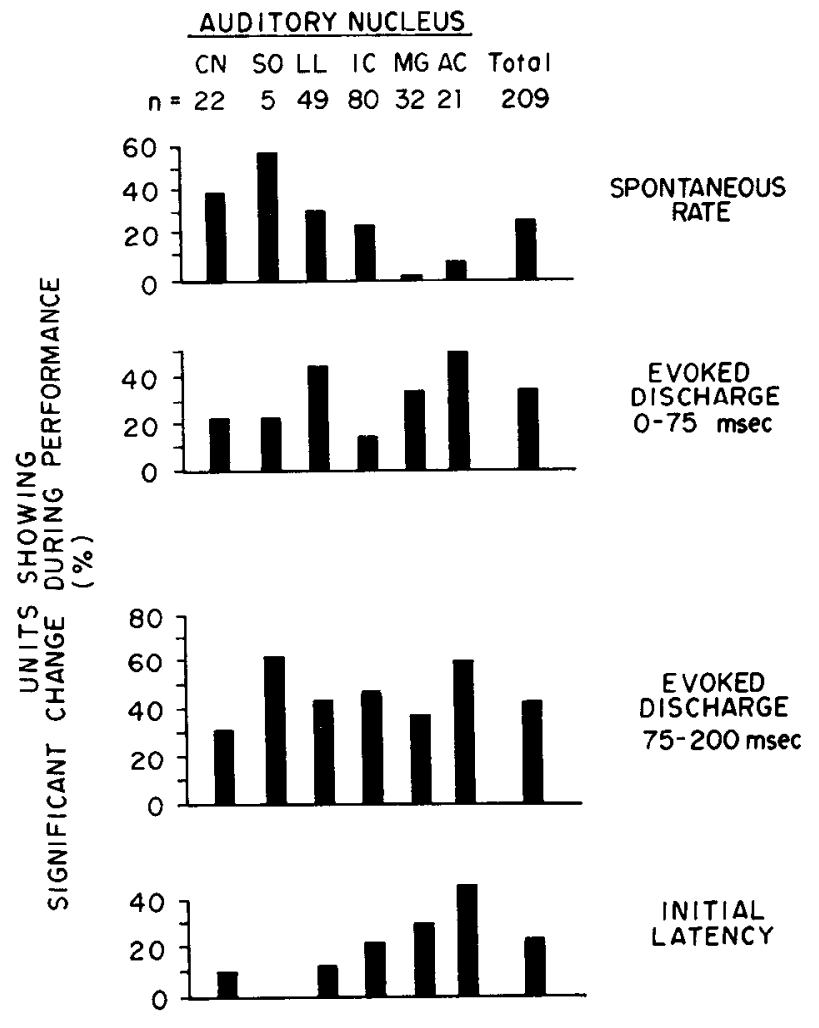

Figure 8. Percentages of auditory units showing significant differences in response characteristics between performance and nonperformance conditions. Abbreviations as in Figure 6. decrease of $1.8 \mathrm{msec}$ during performance across all units. Units showing a significant difference in latency between the two conditions exhibited an average decrease of 3.4 msec during task performance.

Spontaneous rate. Across all units, spontaneous discharge rates during performance increased by about $5 \%$ during reaction time performance, with some stations showing an increase and others showing a decrease. There was a tendency for units at lower auditory stations to show slightly increased spontaneous rates during performance, while those at the medial geniculate and auditory cortex showed either no change or a decrease during performance.

\section{Comparison of unit classes within a nucleus}

Sufficient data to compare the effects of performance on different subdivisions within a given auditory structure were obtained only for the inferior colliculus and lateral lemniscus. The central nucleus and the external capsule of the inferior colliculus were affected similarly by reaction time performance (Ryan and Miller, 1977). The ventral and dorsal nuclei of the lateral lemniscus also showed very similar performance effects, although the consistency and magnitude of changes seen in the ventral nucleus were slightly greater.

We previously reported that inferior colliculus units that exhibited either a phasic onset response or discharge suppression as their principal response pattern were more affected by performance than were other units 


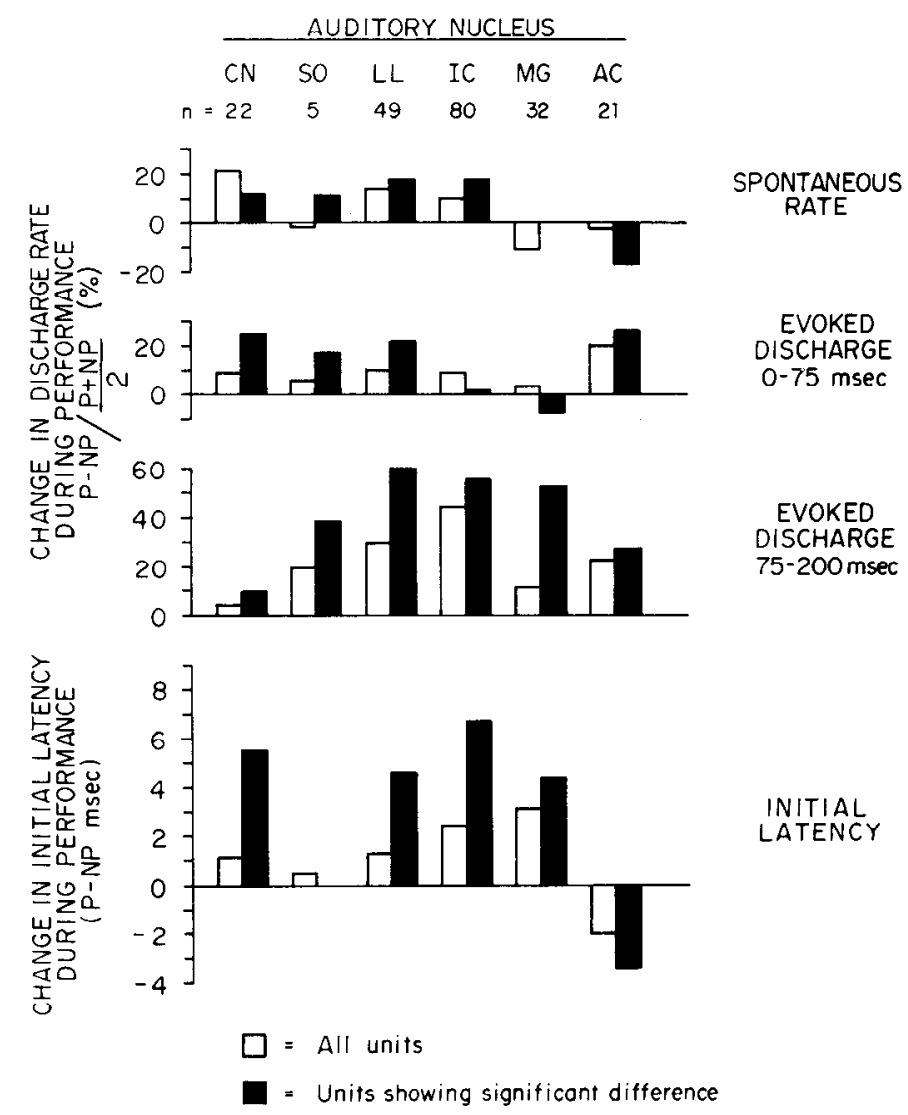

Figure 9. Effect of reaction time performance on unit discharge characteristics at several locations in the central auditory pathway. Discharge rates were normalized by subtracting the nonperformance rate from the performance rate and dividing the resultant figure by the average of the performance and nonperformance rates. Normalized rates more accurately reflect the effects of performance on units with low discharge rates. Abbreviations as in Figure 6.

(Ryan and Miller, 1977). In the present investigation, few units outside of the inferior colliculus exhibited primarily discharge suppression. The unit sample in the lateral lemniscus consisted principally of onset and primary-like response patterns. Figure 10 shows a comparison of performance effects on onset and primary-like units at the lateral lemniscus as well as at the inferior colliculus. In both structures, the effects of performance were much greater on onset units than on primary-like units. This was especially true of the performance-related increases in evoked discharge from 75 to 200 msec after stimulus onset.

\section{Molor units}

The three units recorded in motor structures of the brain stem showed changes in discharge rate during reaction time performance but not during nonperformance (Fig. 11). The unit in the motor nucleus of the trigeminal nerve showed a decrease in discharge rate after stimulus offset in the performance condition. Of the two units in the pyramidal tract, one showed an increase and the other a decrease after stimulus offset during performance. It can be presumed that these

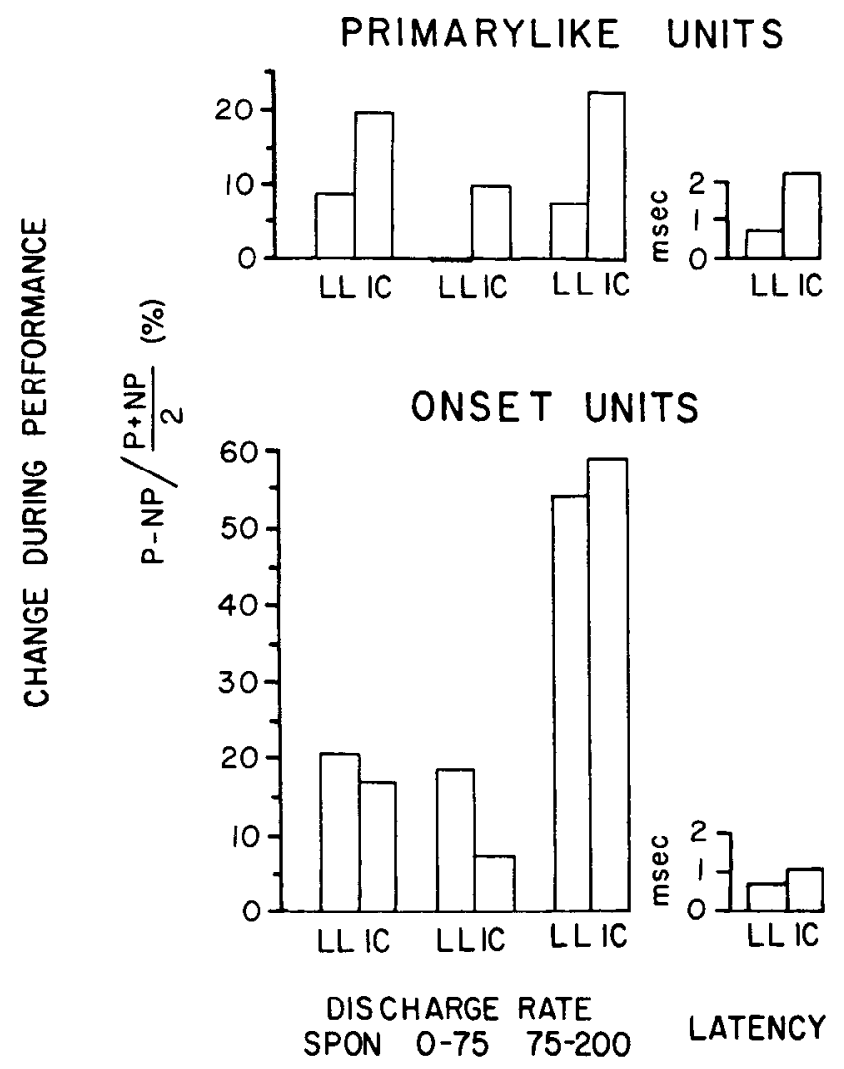

Figure 10. Comparison of the effects of reaction time performance on two different unit response types. Discharge rates were normalized as in Figure 9. In the lateral lemniscus $(L L)$, 22 primary-like and 19 onset units were observed. $I C$, inferior colliculus.
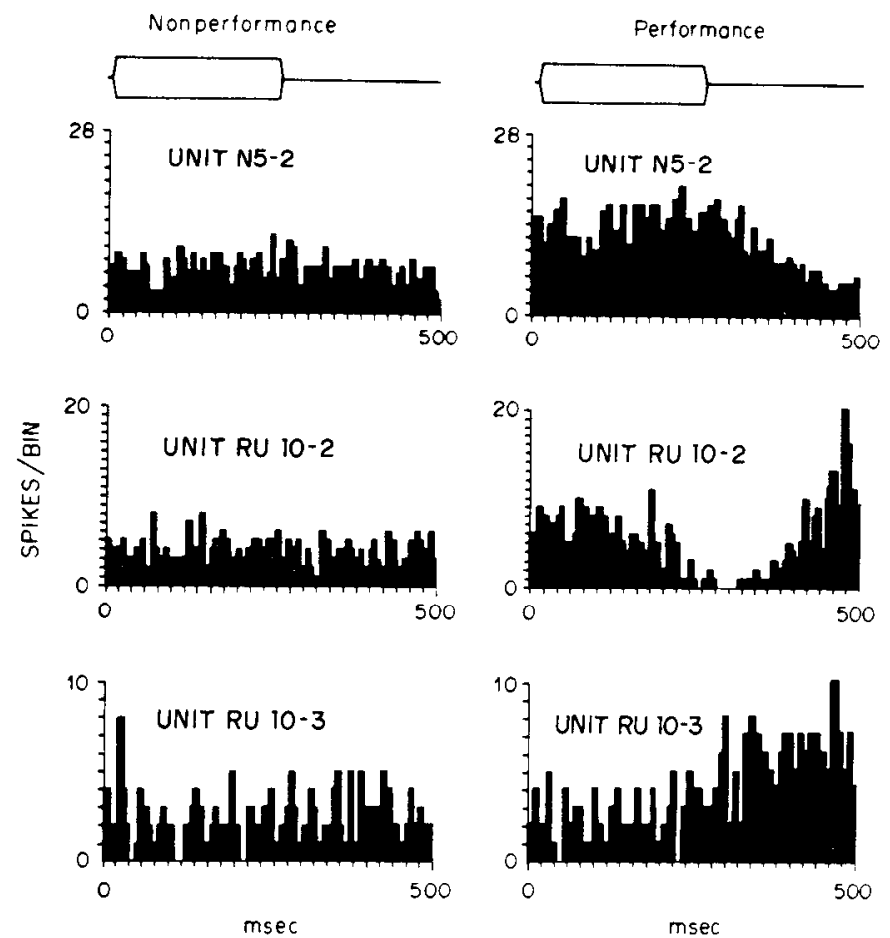

Figure 11. Effect of reaction time performance on units recorded from motor structures in the brain stem; unit N5-2 was in the motor nucleus of the trigeminal nerve, and units RU10-2 and RU10-3 were in the pyramidal tract. 
changes represent stimulus-evoked motor activity associated with reaction time performance.

\section{Discussion}

The results of this study demonstrate that performance-associated changes in the response properties of single units in the central auditory pathway are widespread. A substantial proportion of units displayed response plasticity during task performance not only in auditory cortex but also in subcortical auditory nuclei even at the level of the cochlear nuclei. However, the effects of performance were not comparable at all locations in the pathway.

Differences in the effects of reaction time performance were noted depending on unit location. The clearest example was the finding with regard to latency. Performance was associated with increased initial response latency in subcortical units but decreased latency in cortical units. In addition, performance appeared to influence the spontaneous rates of units more in the lower brain stem auditory nuclei than in midbrain or cortical auditory centers. Conversely, the effects of performance on latency and evoked discharge were quantitatively greater at higher than at lower auditory stations.

Some generalized influences of task performance on unit response parameters were observed. An increase in discharge during the later portion of a stimulus-evoked response was the most common such feature of unit responses during performance. However, some unit classes or even individual units showed quite specific, typically temporally dependent, effects of reaction time performance, including changes in basic response patterns, temporally discrete response suppression (Fig. 7), and selective suppression of subcortical offset responses.

\section{Mechanisms of performance-associated changes}

Several mechanisms have been proposed to account for the effects of attention on auditory perception. Some of these hypotheses may be relevant to the effects of reaction time task performance on auditory neural responses. Skinner and Yingling (1977) have proposed a "thalamic gate" hypothesis, by which sensory input to cortex is controlled at the level of the medial geniculate. The hypothesis predicts that the major effects of attention should be observed at the levels of the medial geniculate and auditory cortex, whereas responses at lower levels should be relatively insensitive. Our data do suggest that there are differences between the effects of task performance on higher and lower auditory nuclei. However, we did not observe a sharp demarcation between the inferior colliculus and the medial geniculate nucleus. Thus, although a gating phenomenon may be relevant to performance effects, it could not be as discretely located as in the Skinner and Yingling model.

A second mechanism, which could account for the enhanced evoked discharge rates that we observed frequently during performance, is a tonic increase in the excitability of auditory units. A nonspecific increase in excitability has been proposed by Woody et al. (1977) to explain the effects of conditioning paradigms on auditory cortical unit responses and by Mountcastle et al. (1981) to account for increases in visually evoked unit activity during attentive fixation. A tonic excitability increase would be expected to increase both spontaneous and evoked discharge rates. However, we observed performance-related increases in stimulus-evoked discharge in the absence of any demonstrable changes in spontaneous rate in the medial geniculate nucleus and auditory cortex. Thus, a general enhancement of unit excitability cannot account for the effects of performance at these levels. It is possible that a general enhancement of auditory unit excitability is employed peripherally, to increase the probability of detection of stimulus onset. This would produce a modest increase in both spontaneous and evoked discharge rates, such as we observed in lower auditory nuclei. It might be expected that an increase in spontaneous rate would be passed along the auditory pathway. A recent study by Woolf et al. (1983) suggests that a successive transfer of spontaneous activity does in fact occur, but only through the level of the inferior colliculus. Presumably, the spontaneous rates of medial geniculate nucleus and auditory cortex are less dependent on auditory afferent input. This finding is consistent with our observations, in which spontaneous rates showed a tendency to increase during performance only up to the colliculus (Figs. 8 and 9).

The marked performance-associated enhancement of evoked discharge at subcortical levels observed after a delay of 50 to $75 \mathrm{msec}$ is consistent with a stimulusevoked descending influence on the subcortical auditory pathway. There is ample evidence of efferent projections which originate in auditory cortex and descend through the entire pathway (Harrison and Howe, 1974; Webster and Aitkin, 1975). These efferent projections could mediate a descending influence. Such efferent activity is typically viewed as inhibitory (Aitkin and Dunlop, 1968; Erulkar et al., 1968). One mechanism that might explain the observed performance-related changes at subcortical levels would involve a performance-enhanced descending inhibitory influence followed by a release from or a decrease in inhibition. Although descending influences on the central auditory pathway could not account for all of the observed effects of performance, they could play a role, especially at the levels of the lateral lemniscus, inferior colliculus, and medial geniculate nucleus.

The observation in auditory cortex of increased discharge at the onset of evoked responses and decreased initial latency during performance, without a concomitant change in spontaneous rate, limits the mechanisms that could mediate performance effects at this level. The lack of effect on spontaneous rate tends to rule out a general increase in unit excitability during performance. Presynaptic facilitation of impulse transmission in selected ascending afferent inputs to cortical cells could accomplish such latency decreases and evoked response enhancement, without increasing spontaneous rates. Woody et al. (1977) observed that classical conditioning produced increases in the responsiveness of auditory cortical neurons to extracellular electrical stimulation but not to intracellular electrical stimulation. This result is consistent with a presynaptic facilitation of evoked auditory responsiveness. Basically, such a model for explaining these effects is one of a class based on the 
premise that the inputs that determine evoked response in auditory cortical cells are at least in part spatially separated from inputs which determine spontaneous rates. Thus, independent facilitation of only one of these cell properties might be feasible. The latency increase of subcortical units, without a corresponding decrease in spontaneous rates, could be accomplished by an analogous inhibitory influence.

Perhaps the most difficult observation to fit within any theoretical framework is the discrepancy between the effects of performance upon initial latency of subcortical units (an increase) versus cortical units (a decrease). Given this rather striking, unexpected difference, we must consider the possibility that the subcortical units sampled in our study did not overlap completely with the population which determined the early evoked response in cortex. Thus, although the average effect of performance upon subcortical unit latency was an increase (see Fig. 9), it should be noted that only a minority of individual subcortical units displayed a significant increase (see Fig. 8). A presynaptic facilitation of input to cortical cells from the remaining subcortical units, which either showed latency reductions during performance or were unaffected, could account for the decrease in cortical unit latency. The observed dependence of subcortical latency shifts upon stimulus intensity, versus the independence of cortical latency shifts, also suggests a basic difference in the determinants of initial latency at these two locations during performance. Cortical changes in latency during performance could be mediated by nonauditory pathways (e.g., diffusely projecting reticular systems), whereas task-related changes in subcortical latency may be more dependent on auditory pathway circuits. This suggestion may find support based on the analysis of performance-related latency changes across a large sample of cortical cells from different areas and layers. Thus, AI cells of layer IV may follow subcortical evoked activity more closely than cells in more superficial layers of this and other areas (Jones and Burton, 1976). Unfortunately, our current sample is inadequate to address this question.

If subcortical latency increases are independent of cortical changes, this in turn suggests that they subserve a different function than the initial detection of stimulus onset. It is possible that a delayed onset response could serve to increase the reliability of onset detection, by providing a confirming neural event, and thus reduce false positive responses in the behavioral task.

Finally, the specific instances of very late discharge suppression, as illustrated in Figure 7, may be related to motor aspects of reaction time performance. They are the only performance-related changes in auditory unit activity that are temporally similar to the performancerelated changes observed in motor units, as illustrated in Figure 11. This interpretation is consistent with the observation that evoked auditory activity is sometimes reduced during movement (Carmel and Starr, 1963; Starr, 1964; Brugge and Merzenich, 1973).

\section{Conclusions}

It is apparent from the discussion above that the effects of reaction time performance that we observed in the monkey central auditory pathway are complex and cannot be explained by any one neural mechanism. It is virtually certain that a number of processes interact in order to produce the observed performance effects. These processes appear to operate with varying degrees of strength at different locations in the central auditory pathway and on different neural populations.

From a functional standpoint, it can be assumed that the changes that occur in auditory signal processing during reaction time performance act to increase the probability of detection of stimulus onset. In fact, the ultimate effects of performance support this assumption. At the cortical level, reaction time performance results in an increase in evoked response magnitude and a reduction in response latency, without an increase in spontaneous rate, producing, in effect, a more sensitive and reliable, yet low-noise, signal detector.

\section{References}

Aitkin, L. M., and C. W. Dunlop (1968) Interplay of excitation and inhibition in the cat medial geniculate body. J. Neurophysiol. 31: 44-61.

Beaton, R., and J. M. Miller (1975) Single cell activity in the auditory cortex of the unanesthetized, behaving monkey: Correlation with stimulus controlled behavior. Brain Res. 100: 543-562.

Bogdanski, D. F., and R. Galambos (1960) Studies of the auditory system with implanted electrodes. In Neural Mechanisms of Auditory and Vestibular Systems, G. L. Rasmussen and W. F. Windle, eds., pp. 143-148, Charles C Thomas, Springfield, IL.

Rrugge, J. F., and M. M. Merzenich (1973) Responses of neurons in auditory cortex of the macaque monkey to monaural and binaural stimulation. J. Neurophysiol. 36: 11381158.

Carmel, P. W., and A. Starr (1963) Acoustic and non-acoustic factors modifying middle-ear muscle activity in waking cats. J. Neurophysiol. 26: 598-616.

Chin, J. H., E. K. Killam, and K. F. Killam (1965) Factors affecting sensory input in the cat: Modification of evoked auditory potential by reticular formation. Electroencephalogr. Clin. Neurophysiol. 18: 567-574.

Erulkar, S. D., J. E. Rose, and P. W. Davies (1956) Single unit activity in the auditory cortex of the cat. Bull. Johns Hopkins Hosp. 99: 55-86.

Erulkar, S. D., R. A. Butler, and G. L. Gerstein (1968) Excitation and inhibition in cochlear nucleus. I. Tone-burse stimulation. J. Neurophysiol. 31: 526-536.

Evans, E. F., and P. G. Nelson (1973) The responses of single neurones in the cochlear nucleus of the cat as a function of their location and the anaesthetic state. Exp. Brain Res. 17: $402-427$.

Harrison, J. M., and M. E. Howe (1974) Anatomy of the descending auditory system (mammalian). In Handbook of Sensory Physiology. Vol. V/1: Auditory System, W. D. Keidel and W. D. Neff, eds., pp. 363-388, Springer-Verlag, New York.

Holstein, S. B., J. S. Buchwald, and J. A. Swafel (1969a) Tone response patterns of auditory nuclei during normal wakefulness, paralysis and anesthesia. Brain Res. 15: 183-499.

Holstein, S. B., J. S. Buchwald, and J. A. Swafel (1969b) Progressive changes in auditory response patterns to repeated tone during normal wakefulness and paralysis. Brain Res. 16: 133-148.

Hubel, D. H., C. O. Henson, A. Rupert, and R. Galambos (1959) "Attention" units in the auditory cortex. Science 129: 12791280 . 
Jones, E. G., and H. Burton (1976) Areal differences in the laminar distribution of thalamic afferents in cortical fields of the insular, parietal and temporal regions of primates. J. Comp. Neurol. 168: 197-248.

Lonsbury-Martin, B. L., and G. K. Martin (1981) Effects of moderately intense sound on auditory sensitivity in rhesus monkeys: Behavioral and neural observations. J. Neurophysiol. 46: 563-586.

Merzenich, M. M., and J. F. Brugge (1973) Representation of the cochlear partition on the superior temporal plane of the macaque monkey. Brain Res. 50: 275-296.

Miller, J. M., and D. Sutton (1976) Techniques for recording single cell activity in the unanesthetized monkey. In Handbook of Auditory and Vestibular Research Methods, C. A. Smith and J. A. Vernon, eds., pp. 226-245, Charles C Thomas, Springfield, IL.

Miller, J. M., D. Sutton, B. Pfingst, A. Ryan, R. Beaton, and G. Gourevitch (1972) Single cell activity in the auditory cortex of rhesus monkeys: Behavioral dependency. Science 177: 449-451.

Miller, J. M., B. E. Pfingst, and A. F. Ryan (1982) Behavioral modification of response characteristics of cells in the auditory system. In Conditioning: Representation of Involved Neural Function, C. D. Woody, ed., pp. 305-362, Academic Press, New York.

Mountcastle, V. B., R. A. Andersen, and B. C. Motter (1981) The influence of attentive fixation upon the excitability of the light-sensitive neurons of the posterior parietal cortex. J. Neurosci. 1: 1218-1235.

Oleson, T. D., J. H. Ashe, and N. M. Weinberger (1975) Modification of auditory and somatosensory system activity during pupillary conditioning in the paralyzed cat. J. Neurophysiol. 38: 1114-1139.

Pandya, D. N., and F. Sanides (1973) Architectonic parcellation of the temporal operculum in rhesus monkey and its projection pattern. Z. Anat. Etwicklungsgesch. 139: 127-161.

Pfingst, B. E., and T. A. O'Connor (1980) A vertical stereotaxic approach to auditory cortex in the unanesthetized monkey. J. Neurosci. Meth. 2: 33-45.

Pfingst, B. E., and T. A. O'Connor (1981) Characteristics of neurons in auditory cortex of monkeys performing a simple auditory task. J. Neurophysiol. 45: 137-155.

Pfingst, B. E., R. Heinz, J. Kimm, and J. M. Miller (1975a) Reaction-time procedure for measurement of hearing. I. Suprathreshold functions. J. Acoust. Soc. Am. 57: 421-430.

Pfingst, B. E., R. Heinz, and J. M. Miller (1975b) Reactiontime procedure for measurement of hearing. II. Threshold functions. J. Acoust. Soc. Am. 57: 431-436.
Pfingst, B. E., T. A. O'Connor, and J. M. Miller (1977) Response plasticity of neurons in auditory cortex of the rhesus monkey. Exp. Brain Res. 29: 393-404.

Ryan, A., and J. Miller (1977) Effects of behavioral performance on single unit firing patterns in inferior colliculus of the rhesus monkey. J. Neurophysiol. 40: 943-956.

Ryan, A., and J. Miller (1978) Single unit responses in the inferior colliculus of the awake and performing rhesus monkey. Exp. Brain Res. 32: 389-408.

Skinner, J. E., and C. D. Yingling (1977) Central gating mechanisms that regulate event related potentials and behavior. A neural model for attention. Prog. Clin. Neurophysiol. 1: $30-69$.

Smith, O. A., K. G. Kastella, and D. C. Randall (1972) A stereotaxic atlas of the brainstem for Macaca mulatta in the sitting position. J. Comp. Neurol. 145: 1-24.

Starr, A. (1964) Influence of motor activity on click-evoked responses in the auditory pathway of waking cats. Exp. Neurol. 10: 191-204.

Stebbins, W. C., and J. Miller (1964) Reaction time as a function of stimulus intensity for the monkey. J. Exp. Anal. Behav. 7: 309-312.

Webster, W. R., and L. M. Aitkin (1975) Central auditory processing. In Handbook of Psychobiology, M. Gazzaniga and C. Blakemore, eds., pp. 325-364, Academic Press, New York.

Wickelgren, W. O. (1968) Effect of state of arousal on clickevoked responses in cats. J. Neurophysiol. 31: 757-768.

Winters, W. D. (1964) Comparison of the averaged cortical and subcortical evoked response to clicks during various stages of wakefulness, slow wave sleep and rhomboencephalic sleep. Electroencephalogr. Clin. Neurophysiol. 17: 234-245.

Woody, C. D. (1977) Changes in activity and excitability of cortical auditory receptive units of the cat as a function of different behavioral states. Ann. N. Y. Acad. Sci. 290: 180199 .

Woody, C. D., J. D. Knispel, T. J. Crow, and P. A. BlackCleworth (1977) Activity and excitability to electrical current of cortical auditory receptive neurons of awake cats as affected by stimulus association. J. Neurophysiol. 39: 10451061.

Woolf, N. K., F. R. Sharp, T. M. Davidson, and A. F. Ryan (1983) Cochlear and middle ear effects on metabolism in the central auditory pathway during silence: A 2-deoxyglucose study. Brain Res. 274: 119-127.

Worden, F. G., and J. T. Marsh (1963) Amplitude changes of auditory potentials evoked at cochlear nucleus during acoustic habituation. Electroencephalogr. Clin. Neurophysiol. 15: $866-881$. 